\title{
Onkologie - eine Erfolgsstory
}

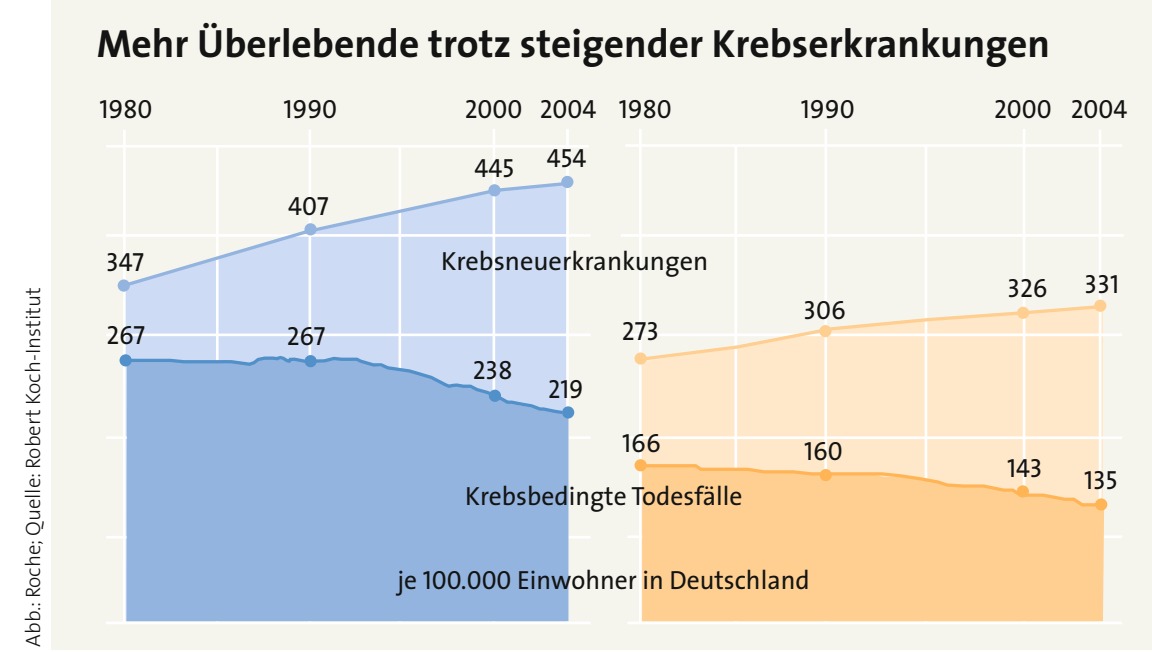

In Deutschland ist seit 1980 ein kontinuierlicher Anstieg der diagnostizierten Krebsneuerkrankungen festzustellen. Dank verbesserter Früherkennung und Fortschritten in der Therapie geht die Zahl der krebsbedingten Todesfälle trotzdem stetig zurück.

\section{ZAHLEN SEIT 1990 STARK GESUNKEN}

\section{Niedrigere Krebssterblichkeit auch in den USA}

Von 1990 bis 2005 ist die Zahl der krebsbedingten Todesfälle in den USA stark zurückgegangen: Bei Männern ist sie um $19,2 \%$ gesunken, verantwortlich ist vor allem der Rückgang bei Todesfällen durch Bronchial-, Prostata- und Kolonkarzinome. Bei Frauen ist die Rate um 11,4\% gesunken, hier vor allem dank einer verminderten Sterblichkeit an Mamma- und kolorektalen Karzinomen. Als wichtige Ursachen für die Abnahme gelten beim kolorektalen Karzinom die frühere Diagnose, beim Mammakarzinom der verminderte Einsatz der Hormonersatztherapie und beim Lungenkrebs der Rückgang der Raucherquote bei Männern. Die schlechten Nachrichten: Immer mehr Amerikaner sterben an Pankreas,- Leber-, Ösophagus- und Hautkrebs. Ebenfalls im Anstieg befindet sich die Zahl der Todesfälle durch Lungenkrebs bei Frauen. CA Cancer J Clin 2009; Juli/August

\section{ALZHEIMER-KRANKHEIT}

\section{Antidementiva können Synkopen auslösen}

Die zur Behandlung der Alzheimer-Demenz eingesetzten Cholinesterasehemmer können die Herzfrequenz herabsetzen und dadurch unter Umständen Synkopen provozieren. In einer Kohortenstudie mit rund 80000 Demenzpatienten wurden diejenigen, die einen Cholinesterasehemmer erhielten - knapp ein Viertel der Patienten -, fast doppelt so häufig wegen Synkopen in ein Krankenhaus ein- gewiesen wie Patienten ohne eine solche Therapie (31,5 vs. 18,6 pro 1000 Patientenjahre). Auch Bradykardien, Schrittmacherimplantationen und Hüftfrakturen wurden häufiger registriert als in der Kontrollgruppe. Die Studienautoren raten deshalb, den Nutzen der Cholinesterasehemmer bei Patienten mit früheren Synkopen oder Bradykardie sorgfältig zu prüfen. Arch Intern Med 2009;169:867-73

\section{THERAPIENEBENWIRKUNGEN}

\section{Wie ein Krebspatient fast im Gefängnis landete}

Dass eine onkologische Therapie reich an Nebenwirkungen ist, ist eine Binsenweisheit. Aber dass zu diesen Nebenwirkungen auch Probleme mit der Einwanderungsbehörde der USA gehören können, steht wohl auf keinem Beipackzettel.

Ein 62-Jähriger aus Singapur wurde wegen Tumoren im Kopf- und Nackenbereich mit Capecitabin behandelt und entwickelte ein leichtes Hand-Fuß-Syndrom. Der Patient wollte in die USA einreisen und sollte dort bei der Einreisebehörde seine Fingerabdrücke hinterlassen. Diese waren jedoch bedingt durch das HandFuß-Syndrom verschwunden. Nach einem mehrstündigen Verhör waren die US-Behörden endlich davon überzeugt, dass er kein Terrorist sei und er durfte ins Land. Er erhielt noch den guten Rat, auf zukünftige Reisen ein Schreiben seines Onkologen mitzuführen, in dem das Fehlen der Fingerabdrücke erklärt werde. Annals of Oncology, DOl: 10.1093/annonc/mdp278

\section{DEPRESSION NACH INFARKT}

\section{Der Tod der traurigen Herzen}

Patienten, die zwei bis drei Wochen nach Infarkt unter einer Depression leiden, haben eine wesentlich höhere Sterblichkeit in den folgenden sieben Jahren als Patienten mit stabilerer Psyche. Damit bestätigte die SADHART-Studie erstmals die prognostische Bedeutung einer Postinfarkt-Depression auch im Langzeitverlauf. Die Prognose ist umso ungünstiger, je schwerer die Depression nach dem koronaren Ereignis ist. Bei einem Score über 18 auf der Hamilton-Depression-RatingSkala ist die Mortalität innerhalb der nächsten sieben Jahre doppelt so hoch wie bei anderen Infarktpatienten.

Jahrestagung der American Psychiatric Association, San Francisco, 21. Mai 2009 